\section{O papel dos estados na política de saúde mental no Brasil}

\author{
The role of States in mental health \\ policy in Brazil
}

Aline Gabriela Simon 1

Tatiana Wargas de Faria Baptista 1
1 Escola Nacional de Saúde Pública Sergio Arouca, Fundação Oswaldo Cruz, Rio de Janeiro, Brasil.

Correspondência A. G. Simon

Departamento de Administração e Planejamento em Saúde, Escola Nacional de Saúde Pública Sergio Arouca, Fundação Oswaldo Cruz. Rua Leopoldo Bulhões 1480, 70 andar, Rio de Janeiro, $R J$ 21041-210, Brasil. linegabriela@yahoo.com.br

\section{Abstract}

This article examines the role of States in mental health policy in Brazil from 1990 to 2009. The methods included the use of an electronic questionnaire on State coordination of mental health in 24 Brazilian States, document review, and analysis based on official data. The results showed that the States use various strategies and tools to conduct mental health policy, especially in monitoring and services delivery. However, the study showed that States have not developed systematic approaches to planning, coordination with other levels of government, or coordination of care. Funding poses a major challenge for management of mental health at the State level. The study suggests that overcoming the challenges in mental health policy depends on the relationship between the Federal government, States, and Municipalities in drafting policies to address each region's specific needs, the promotion of participatory planning, and investment in the sector.

Mental Health; Health Policy; Health Management

\section{Introdução}

A configuração do Sistema Único de Saúde (SUS) e a instituição de seus princípios representaram a consolidação de um processo de lutas pela democratização do acesso à saúde, impulsionado pelo movimento da reforma sanitária. No entanto, o formato da descentralização assumido na Constituição de 1988 e na Lei Orgânica da Saúde 1 implicou mudanças no papel atribuído aos três entes federativos, sendo a esfera estadual preterida neste modelo, o qual enfatizou, principalmente, o papel dos municípios 2.

Paralelamente, na área de saúde mental, consolidou-se, no Brasil, o movimento da reforma psiquiátrica, que mobilizou um importante debate em torno do resgate da cidadania dos usuários dos serviços de saúde mental e da transformação e superação da estrutura asilar. Sua operacionalização em política nacional na década de 1990, com a criação da Coordenação Nacional de Saúde Mental (CORSAM), inaugurou um novo marco na produção normativa em saúde mental, por meio da apresentação de portarias com o objetivo de propor mudanças no modelo assistencial ${ }^{3}$. Também a aprovação da Lei $n^{o}$. 10.216/2001, que redireciona o modelo assistencial em saúde mental 4, após um longo processo de tramitação no Congresso, viabilizou a criação de políticas na área e a mobilização de profissionais, instituições e sociedade para um 
novo olhar social e de atenção aos usuários dos serviços de saúde mental.

A criação de uma política específica de saúde mental deve ser compreendida no contexto do SUS. O desenho da política nacional de saúde mental também privilegiou a municipalização dos serviços substitutivos 5 , seguindo a descentralização da saúde em geral. Entretanto, em face do perfil dos municípios do país, dos quais mais de $75 \%$ têm menos de 20 mil habitantes, e a complexidade na formulação de um modelo de atenção em saúde mental, depreende-se que a participação dos estados torna-se fundamental na formulação e adaptação regional da política, na construção de uma rede substitutiva de serviços, na coordenação das ações no território, no combate às desigualdades e na melhoria das condições locais com fortalecimento da capacidade institucional nos municípios, principalmente naquelas regiões onde existem verdadeiros vazios assistenciais.

Além disso, não obstante a importância de uma política nacional para a direcionalidade do processo político, esta não deve implicar a adoção de padrões e modelos uniformes em todo o territorial nacional. Assim, torna-se necessária a construção de políticas direcionadas para as especificidades locorregionais.

A partir disso, algumas questões se apresentam: De que forma os estados têm desempenhado seu papel na política de saúde mental? Eles têm sido capazes de diversificar as estratégias e instrumentos preconizados pela política nacional, de modo a atender às especificidades regionais e a mudança do modelo assistencial em saúde mental? Eles têm desempenhado função relevante na organização do sistema de serviços nessa área? Que recursos os estados possuem para exercer esse papel? Quais as suas principais dificuldades?

Este é um tema de grande importância na atualidade. Nos últimos anos, o foco principal dos estudos tem sido as experiências municipais ou a condução da política nacional; na área de saúde mental, são escassos os estudos que evidenciam o que tem sido o esforço do ente estadual na construção da política de saúde mental. Diante disso, este estudo busca preencher essa lacuna e contribuir para uma reflexão acerca dos desafios dos estados na condução da política.

Em adição, o diálogo com a literatura internacional reforça tal esforço ao indicar a necessidade de implantação de políticas coordenadas, planos e programas de saúde mental; articulação com setores não-governamentais; ampliação dos serviços para toda a população; e estabelecimento de um fluxo financeiro efetivo para o desenvolvimento das ações de saúde mental na comunidade, principalmente nos países de média e baixa renda 6,7,8,9.

Estas propostas, embora referidas em nível global, possibilitam uma interface com estudos nacionais para o direcionamento do papel dos estados na política de saúde mental no Brasil.

Este artigo analisa o papel dos estados na política de saúde mental brasileira, considerando as diretrizes nacionais e as estratégias adotadas pelos estados na condução desta política. Procura-se identificar as estratégias utilizadas pelos estados na condução da política de saúde mental, reconhecer iniciativas estaduais de conformação de modelos assistenciais que não estão previstos nas normativas federais, discutir os desafios e analisar as implicações dos estados na mudança do modelo.

\section{Metodologia}

As estratégias utilizadas pelos estados na condução da política de saúde mental foram analisadas levando-se em consideração seu papel segundo as macrofunções gestoras do Estado na saúde. Em um país federativo, em que a autoridade política do Estado e o exercício do poder autônomo são repartidos em múltiplos centros soberanos e coordenados entre si 10, essas funções representariam atribuições comuns das três esferas de governo, caracterizando um dado modelo de intervenção estatal na saúde.

Alguns autores identificam diferentes macrofunções gestoras na saúde 11,12. Nesta pesquisa, adotamos como referencial o estudo de Lima et al. 13, adequando-o à realidade da saúde mental (Tabela 1). Outro referencial de análise partiu do trabalho de Borges 14 sobre a trajetória da política nacional de saúde mental (Tabela 2). A análise da política possibilitou um balanço do papel dos estados na produção normativa federal na década de 1990, com a atualização do quadro a partir de 2005 .

O presente estudo abrange o período de 1990 a 2009 e visou a identificar as estratégias da política de saúde mental adotadas no âmbito dos estados. Para uma análise mais aprofundada do papel dos estados, seria necessário o emprego de outras técnicas de pesquisa e a análise específica de cada realidade estadual.

Foram adotadas as seguintes estratégias metodológicas: análise de documentos oficiais (documentos, portarias, leis, relatórios das conferências, pesquisa aos sites das secretarias de saúde), análise de base de dados oficiais e aplicação de questionário eletrônico, que foi respondido pelas coordenações estaduais de saúde mental de 24 estados brasileiros. 
Os estados participantes desta pesquisa foram:

- Rio de Janeiro, Minas Gerais e São Paulo, na Região Sudeste;

- Rio Grande do Sul, Paraná e Santa Catarina, na Região Sul;

- Maranhão, Rio Grande do Norte, Piauí, Paraíba, Alagoas, Ceará, Pernambuco e Sergipe, na Região Nordeste;

- Distrito Federal, Goiás, Mato Grosso, Mato Grosso do Sul, na Região Centro-oeste;

- Acre, Amazonas, Amapá, Pará, Roraima e Tocantins, na Região Norte.
Todos os estados participantes da pesquisa assinaram o termo de consentimento livre e esclarecido, cumprindo as exigências da Resolução $n^{o}$. 196/96 do Conselho Nacional de Saúde (CNS).

\section{O papel dos estados nas normativas e diretrizes de saúde mental}

A política nacional de saúde mental iniciou uma nova trajetória no ano de 1991 com a criação da CORSAM. O cenário era de grande articulação de

As macrofunções gestoras do estado na política de saúde mental.

\begin{tabular}{|c|c|c|}
\hline Macrofunções & Atributos na política de saúde & Especificidades da política de saúde mental \\
\hline $\begin{array}{l}\text { Formulação de políticas e } \\
\text { planejamento }\end{array}$ & $\begin{array}{l}\text { Definição das regiões de saúde; repartição de } \\
\text { responsabilidades entre distintas esferas de governo } \\
\text { na gestão do sistema e serviços das regiões de saúde; } \\
\text { identificação das necessidades de saúde das populações }\end{array}$ & $\begin{array}{c}\text { Estruturação das coordenações de saúde mental; } \\
\text { características do plano de saúde mental; regionalização e } \\
\text { divisão de responsabilidades entre as esferas de governo } \\
\text { na gestão do sistema de saúde mental; identificação das } \\
\text { necessidades de saúde mental }\end{array}$ \\
\hline Financiamento & $\begin{array}{l}\text { Participação no financiamento do SUS; alocação regional } \\
\text { de recursos próprios para o financiamento da saúde; } \\
\text { promoção da programação pactuada e integrada da } \\
\text { assistência à saúde; mobilização de recursos e definição } \\
\text { de plano de investimentos }\end{array}$ & $\begin{array}{l}\text { Participação no financiamento do SUS (percentual de } \\
\text { recursos próprios destinados às ações de saúde mental); } \\
\text { critérios utilizados para a alocação de recursos próprios } \\
\text { destinados às ações de saúde mental; formas e critérios } \\
\text { utilizados para definir os montantes de recursos a serem } \\
\text { transferidos do Ministério da Saúde para os municípios }\end{array}$ \\
\hline Coordenação federativa & $\begin{array}{l}\text { Implantação de instâncias de gestão regional em saúde; } \\
\text { regulamentação da gestão do sistema e serviços nas } \\
\text { regiões de saúde; promoção da pactuação e formalização } \\
\text { de compromissos entre entes governamentais em âmbito } \\
\text { regional; fortalecimento da capacidade institucional dos } \\
\text { municípios e outros órgãos de gestão descentralizada do } \\
\text { SUS em âmbito regional }\end{array}$ & $\begin{array}{c}\text { Arranjos adotados para a gestão em saúde mental } \\
\text { (comissões e colegiados de saúde mental); instrumentos } \\
\text { legais e normativos de pactuação e formalização } \\
\text { de compromissos em saúde mental; mecanismos e } \\
\text { instrumentos utilizados para o fortalecimento dos } \\
\text { municípios (visitas, supervisões, treinamentos) }\end{array}$ \\
\hline Coordenação da atenção & $\begin{array}{l}\text { Implantação de mecanismos de referência e } \\
\text { contrarreferência entre serviços nas regiões de saúde; } \\
\text { regulamentação da prestação de serviços nas regiões } \\
\text { de saúde; contratualização de serviços de abrangência } \\
\text { regional }\end{array}$ & $\begin{array}{c}\text { Reestruturação da assistência em saúde mental; } \\
\text { mecanismos de organização do sistema de referência } \\
\text { e contrarreferência entre serviços de saúde mental; } \\
\text { intersetorialidade; participação social }\end{array}$ \\
\hline $\begin{array}{l}\text { Monitoramento e } \\
\text { acompanhamento }\end{array}$ & $\begin{array}{l}\text { Existência de serviços voltados para o controle, avaliação, } \\
\text { monitoramento e auditoria do sistema e serviços que } \\
\text { conformam as regiões de saúde; acompanhamento e } \\
\text { avaliação de indicadores com base nos objetivos e metas } \\
\text { propostos no plano regional de saúde, pactos e contratos } \\
\text { envolvendo gestores e prestadores de serviços }\end{array}$ & $\begin{array}{c}\text { Mecanismos e instrumentos utilizados para o } \\
\text { acompanhamento e monitoramento dos serviços } \\
\text { de saúde mental; acompanhamento e avaliação de } \\
\text { indicadores dos serviços de saúde mental }\end{array}$ \\
\hline Prestação de serviços & $\begin{array}{l}\text { Prestação de serviços de referência regional por órgão da } \\
\qquad \text { Administração Direta ou Indireta }\end{array}$ & $\begin{array}{l}\text { Prestação de ações e serviços, por meio de órgãos da } \\
\text { Administração Direta ou Indireta vinculados à Secretaria } \\
\text { Estadual de Saúde, nos diversos campos da atenção à } \\
\text { saúde mental }\end{array}$ \\
\hline
\end{tabular}

Fonte: Adaptado de Lima et al. ${ }^{13}$. 
Momentos da Política Nacional de Saúde Mental e o papel dos estados (1990-2009).

$\begin{array}{llccc}\text { Momentos } & \text { Caracterização } & \text { Contexto da Política } & \text { Principais eventos } & \text { Principais } \\ \text { da Política } & & \text { Nacional de Saúde } & \text { da Saúde Mental } & \text { portarias nacionais } \\ \text { Nacional de } & & & \\ \text { Saúde Mental } & & & \end{array}$

\begin{tabular}{|c|c|c|c|c|c|}
\hline & & & & & \\
\hline (1991-1996) & $\begin{array}{c}\text { de uma política } \\
\text { nacional de saúde } \\
\text { mental } \\
\text { Criação da CORSAM } \\
\text { Articulação com atores } \\
\text { diversos }\end{array}$ & $\begin{array}{l}\text { Nacional de Saúde } \\
\text { NOB } 91 \text { e NOB 92: } \\
\text { ênfase municipalista } \\
\text { X Conferência } \\
\text { Nacional de Saúde } \\
\text { NOB 96: PAB e início } \\
\text { de uma retomada } \\
\text { das atribuições do } \\
\text { estado }\end{array}$ & $\begin{array}{c}\text { CNSM } \\
\text { Criação do } \\
\text { Colegiado de } \\
\text { Coordenadores } \\
\text { Estaduais de Saúde } \\
\text { Mental }\end{array}$ & $\begin{array}{c}\text { 189/91: ênfase na } \\
\text { desospitalização e } \\
\text { qualificação } \\
\text { Portaria SNAS n. } \\
\text { 224/92: regulamenta } \\
\text { o funcionamento de } \\
\text { todos os serviços de } \\
\text { saúde mental } \\
\text { Portaria SAS n. } \\
\text { 145/94: define a } \\
\text { criação de Grupos } \\
\text { de Avaliação da } \\
\text { Assistência Psiquiátrica } \\
\text { (GAP) } \\
\text { Portaria SAS n. 15/96: } \\
\text { ratifica a proibição de } \\
\text { novas internações em } \\
\text { hospitais psiquiátricos }\end{array}$ & $\begin{array}{l}\text { Conselho Estadual e Participação no } \\
\text { Colegiado de Coordenadores Estaduais } \\
\text { Coordenação da atenção: cadastro } \\
\text { dos serviços de Saúde Mental e } \\
\text { competência exclusiva para emissão } \\
\text { de AlH } \\
\text { Monitoramento: supervisão dos } \\
\text { prestadores }\end{array}$ \\
\hline $\begin{array}{l}\text { Latência } \\
\text { (1997-1999) }\end{array}$ & $\begin{array}{l}\text { Projeto político } \\
\text { subentendido } \\
\text { Saída da área técnica } \\
\text { de saúde mental da } \\
\text { SAS } \\
\text { Coordenação técnica, } \\
\text { sem peso político } \\
\text { Queda da produção } \\
\text { normativa federal }\end{array}$ & $\begin{array}{l}\text { Retomada do debate } \\
\text { sobre o papel dos } \\
\text { estados iniciada com } \\
\text { a NOB } 96 \\
\text { Aprovação da } \\
\text { Política Nacional } \\
\text { de Medicamentos } \\
\text { (Portaria GM } n^{\circ} \text {. } \\
\text { 3.916/1998) }\end{array}$ & & $\begin{array}{l}\text { Portaria GM n. } \\
\text { 1.077/99: implanta } \\
\text { o Programa para } \\
\text { a Aquisição dos } \\
\text { Medicamentos } \\
\text { Essenciais para a área } \\
\text { de saúde mental }\end{array}$ & $\begin{array}{c}\text { Coordenação federativa: } \\
\text { gerenciamento do programa para } \\
\text { aquisição de medicamentos essenciais } \\
\text { Financiamento: participação no } \\
\text { financiamento do programa para } \\
\text { aquisição de medicamentos essenciais } \\
\text { em saúde mental }\end{array}$ \\
\hline $\begin{array}{l}\text { Retomada } \\
\text { (2000-2001) }\end{array}$ & $\begin{array}{l}\text { Nova estrutura } \\
\text { ministerial e retorno da } \\
\text { área técnica de saúde } \\
\text { mental para a SAS } \\
\text { Nova coordenação } \\
\text { Novas linhas de } \\
\text { atuação }\end{array}$ & $\begin{array}{l}\text { XI Conferência } \\
\text { Nacional de Saúde } \\
\text { NOAS } 2001 \text { e } 2002 \text { : } \\
\text { regionalização e } \\
\text { ênfase nos estados }\end{array}$ & $\begin{array}{c}\text { III CNSM } \\
\text { Lei n. } 10.216 / 2001\end{array}$ & $\begin{array}{c}\text { Portaria GM n. } \\
\text { 106/00: cria os } \\
\text { Serviços Residenciais } \\
\text { Terapêuticos } \\
\text { Portaria GM n. } \\
\text { 799/00: institui o } \\
\text { Programa Permanente } \\
\text { de Organização e } \\
\text { Acompanhamento das } \\
\text { Ações Assistenciais em } \\
\text { Saúde Mental }\end{array}$ & $\begin{array}{c}\text { Coordenação da atenção: estruturação } \\
\text { de Grupo Técnico de Organização } \\
\text { e Acompanhamento das Ações } \\
\text { Assistenciais em Saúde Mental. } \\
\text { Coordenação federativa: articular as } \\
\text { ações que serão desenvolvidas pelos } \\
\text { Grupos Técnicos dos municípios em } \\
\text { Gestão Plena do Sistema Municipal. } \\
\text { Acompanhamento e monitoramento: } \\
\text { estabelecer, com o apoio técnico } \\
\text { do Ministério da Saúde, rotinas } \\
\text { de acompanhamento, supervisão, } \\
\text { controle e avaliação dos Serviços } \\
\text { Residenciais Terapêuticos em } \\
\text { saúde mental } \\
\text { Emitir relatórios técnicos e } \\
\text { propor medidas corretivas e de } \\
\text { aperfeiçoamento do sistema de } \\
\text { assistência }\end{array}$ \\
\hline
\end{tabular}

(continua) 
Tabela 2 (continuação)

\begin{tabular}{|c|c|c|c|c|c|}
\hline $\begin{array}{l}\text { Momentos } \\
\text { da Política } \\
\text { Nacional de } \\
\text { Saúde Mental }\end{array}$ & Caracterização & $\begin{array}{c}\text { Contexto da } \\
\text { Política Nacional } \\
\text { de Saúde }\end{array}$ & $\begin{array}{l}\text { Principais eventos } \\
\text { da Saúde Mental }\end{array}$ & $\begin{array}{l}\text { Principais } \\
\text { portarias nacionais }\end{array}$ & $\begin{array}{l}\text { Principais atribuições dos } \\
\text { estados por macrofunções }\end{array}$ \\
\hline $\begin{array}{l}\text { Expansão } \\
(2002-2009)\end{array}$ & $\begin{array}{c}\text { Expansão do } \\
\text { modelo de atenção, } \\
\text { diversificando as linhas } \\
\text { de atuação }\end{array}$ & $\begin{array}{l}\text { XII Conferência } \\
\text { Nacional de Saúde } \\
\text { Pacto de Gestão } \\
\text { 2006: Plano Diretor } \\
\text { de Regionalização } \\
\text { (PDR) } \\
\text { XIII Conferência } \\
\text { Nacional de Saúde }\end{array}$ & $\begin{array}{l}\text { Retomada do } \\
\text { Colegiado de } \\
\text { Coordenadores } \\
\text { Estaduais }\end{array}$ & $\begin{array}{l}\text { Portaria GM n. } \\
\text { 251/02: regulamenta o } \\
\text { Programa Nacional de } \\
\text { Avaliação do Sistema } \\
\text { Hospitalar/Psiquiatria } \\
\text { (PNASH) } \\
\text { Portaria GM n. } \\
\text { 336/02: regulamenta } \\
\text { a criação de novas } \\
\text { modalidades de CAPS } \\
\text { (infantil, ad, I, II e III) } \\
\text { Portaria GM n. } \\
\text { 2.077/03: regulamenta } \\
\text { o Programa de Volta } \\
\text { Para Casa (PDV) } \\
\text { Portaria GM n. } \\
\text { 52/04: institui o } \\
\text { Programa Anual de } \\
\text { Reestruturação da } \\
\text { Assistência Psiquiátrica } \\
\text { Hospitalar no SUS } \\
\text { (PRH) } \\
\text { Portaria n. } 2.197 / 04: \\
\text { institui o Programa de } \\
\text { Atenção Integral aos } \\
\text { Usuários de Álcool e } \\
\text { Drogas } \\
\text { Portaria GM n. } \\
\text { 1.612/05: aprova } \\
\text { as normas de } \\
\text { funcionamento e } \\
\text { credenciamento dos } \\
\text { Serviços Hospitalares } \\
\text { de Referência para a } \\
\text { Atenção Integral aos } \\
\text { Usuários de Álcool e } \\
\text { outras Drogas } \\
\text { (SHR-ad) }\end{array}$ & $\begin{array}{l}\text { Formulação: desenvolver de } \\
\text { forma articulada com os demais } \\
\text { entes, programas de saúde mental } \\
\text { destinados aos usuários de álcool e } \\
\text { drogas; estabelecer o planejamento } \\
\text { da rede e a distribuição regional dos } \\
\text { CAPSad } \\
\text { Coordenação federativa: assessorar } \\
\text { os municípios no processo de } \\
\text { implementação dos programas } \\
\text { destinados aos usuários de álcool e } \\
\text { drogas } \\
\text { Coordenação da atenção: estruturar } \\
\text { a implementação de CAPSad e } \\
\text { estabelecer os fluxos de referência e } \\
\text { contrarreferência entre estes serviços } \\
\text { Acompanhamento e monitoramento: } \\
\text { avaliar os hospitais psiquiátricos } \\
\text { integrantes do SUS por meio } \\
\text { do PNASH/Psiquiatria pelos } \\
\text { Grupos Técnicos de Organização } \\
\text { e Acompanhamento das Ações } \\
\text { Assistenciais em Saúde Mental; } \\
\text { efetuar o acompanhamento, controle, } \\
\text { avaliação e auditoria dos SHR-ad } \\
\text { Prestação de ação e serviços de } \\
\text { saúde: definir ações de vigilância } \\
\text { epidemiológica e sanitária no que se } \\
\text { refere aos transtornos causados pelo } \\
\text { uso prejudicial de álcool e drogas } \\
\text { Financiamento: alocar recursos } \\
\text { financeiros próprios para o } \\
\text { desenvolvimento de programas de } \\
\text { saúde mental destinados aos usuários } \\
\text { de }\end{array}$ \\
\hline
\end{tabular}

AlH: Autorizações de Internação Hospitalar; CAPS: Centros de Atenção Psicossocial; CAPSad: Centros de Atenção Psicossocial, Álcool e Drogas; CNSM: Conferência Nacional de Saúde Mental; CORSAM: Coordenação Nacional de Saúde Mental; NOAS: Norma Operacional da Assistência à Saúde; NOB: Norma Operacional Básica; PAB: Piso de Atenção Básica; SAS: Secretaria de Atenção à Saúde; SNAS: Secretaria Nacional de Assistência à Saúde. Fonte: Elaborado com base nos momentos da política nacional identificados por Borges 14 e na revisão dos documentos da política nacional de saúde mental de todo o período para a caracterização da atribuição prevista para os estados. 
atores na discussão dessa política. Desde 1989, uma série de debates e audiências promovidos em torno do Projeto de Lei $n^{\circ}$. 3.657/89 abria para a CORSAM um novo canal de interlocução e aglutinação de força política com o legislativo; em 1992, realizava-se a II Conferência Nacional de Saúde Mental (CNSM), expandindo o debate com os atores de todo Brasil. Além disso, a atuação da coordenação na organização da assistência hospitalar promoveu estratégias de desospitalização, fundamentadas em ações de avaliação e acreditação hospitalar 3 .

No âmbito dos estados, em sua grande maioria, a política de saúde mental ainda se apresentava bastante incipiente; poucos estados haviam estabelecido, nesse período, coordenações e planos de saúde mental. Mas é também desse mesmo período a criação do Colegiado dos Coordenadores Estaduais de Saúde Mental, que se constituiu em importante mecanismo de articulação e negociação intergovernamental, contribuindo para a construção de consensos em torno da política 14 .

A criação do Colegiado dos Coordenadores Estaduais de Saúde Mental possibilitou a construção, de forma participativa, das normativas nacionais apresentadas nesse período. No entanto, ainda havia certa indefinição quanto ao papel dos estados na condução da política, refletindo o peso do debate municipalista da saúde nos primeiros anos da década (Tabela 2).

Até o ano de 1996, a CORSAM manteve-se sob a mesma coordenação e implementou um curso no desenvolvimento da política nacional, enfatizando as seguintes funções: de formulação e planejamento, com a definição de um programa, plano ou projeto assistencial para a população remanescente de hospitais psiquiátricos; de monitoramento e acompanhamento, com a criação dos Grupos de Avaliação da Assistência Psiquiátrica (GAP); de coordenação da atenção, constituindo políticas de desospitalização, avaliação e acreditação da assistência hospitalar, com a responsabilidade pela complementação das normas técnicas e cadastramento dos serviços de saúde mental nos sistemas de informação do SUS. Tais políticas estimularam a adesão dos estados, mas não levou à definição de atribuições mais específicas para o nível estadual.

Ressalte-se que o relatório da II CNSM já havia definido um conteúdo mais específico em relação ao papel dos estados, mas as condições institucionais e políticas para o desenvolvimento dessas atribuições ainda não estavam dadas. Ainda assim, deve-se considerar a importante atuação de alguns estados na formulação da política desde a década de 1980 e a aprovação de leis estaduais neste período, mesmo antes da aprovação da lei nacional (Ceará, Pernambuco, Rio Grande do Norte, Distrito Federal, Minas Gerais, Paraná, Espírito Santo e Rio Grande do Sul). Deve-se destacar também o papel gestor dos estados no controle dos leitos psiquiátricos.

Entre 1997 a 1999 houve queda da produção normativa nacional. Em 1997, ocorreu uma mudança na estrutura do Ministério da Saúde, com a formação de novas Secretarias. Nesse período, a Secretaria que abrigava a CORSAM sofreu um enfraquecimento, desarticulando-se como estrutura de formulação política, o que resultou no vazio de portarias 14 .

No contexto mais geral da política de saúde, iniciou-se a retomada do debate sobre o papel dos estados, principalmente a partir da NOB 96. Uma nova portaria (PT/GM no $n^{\circ}$ 1.077/99) definiu que os estados passariam a gerenciar não só o programa para aquisição de medicamentos essenciais em saúde mental, como também os recursos financeiros destinados aos municípios sem rede pública de atenção ambulatorial nesse setor. Assim, a partir de 2000, com a edição da NOAS/2001, os estados assumiram papel mais ativo no cenário da política de saúde mental (Tabela 2).

As portarias do período definem atribuições para os estados no acompanhamento, supervisão e coordenação dos serviços, com a estruturação dos Grupos Técnicos de Organização e Acompanhamento das ações assistenciais em saúde mental em âmbito estadual (PT/GM $n^{\circ}$. 799/00) e da supervisão dos Serviços Residenciais Terapêuticos (PT/GM n $n^{\circ}$. 1.220/00). Também neste período foi aprovada a Lei $n^{\circ}$. 10.216/2001 e realizou-se a III CNSM.

O relatório da III CNSM foi o mais completo em termos de atribuições dos estados na política de saúde mental, prevendo a participação destes na elaboração de leis e planos, no financiamento e apoio ao processo de municipalização dos serviços, no acompanhamento e supervisão dos serviços, na atuação na Comissão Intergestores Bipartite (CIB) e na articulação com as instâncias de controle social.

A partir de 2002, a política de saúde mental iniciou algumas inflexões importantes, intensificando ações condizentes com as diretrizes da Conferência e determinações da lei federal 14.

Em 2003, é criado o Colegiado de Coordenadores de Saúde Mental (CCSM), sendo composto, naquele momento, por coordenadores de saúde mental dos 27 estados, das capitais e grandes cidades, além de contar com representantes e consultores do Ministério da Saúde, do Conselho Nacional de Secretários de Saúde (CONASS) e do Conselho Nacional de Secretários Municipais de Saúde (CONASEMS). Desde então, este colegiado 
tem a tarefa de discutir os desafios e avanços do processo de mudança do modelo assistencial em saúde mental nos estados brasileiros 15 .

A maior parte das portarias desse período traz atribuições específicas em relação ao papel dos estados na política (Tabela 2), principalmente nas funções de coordenação da atenção, coordenação federativa e monitoramento e acompanhamento em saúde mental. Também se observa a definição de maior participação dos estados na formulação de políticas de atenção voltadas aos usuários de álcool e outras drogas, como o planejamento da rede e distribuição regional de Centros de Atenção Psicossocial, Álcool e Drogas (CAPSad). Contudo, a definição da participação dos estados no financiamento das ações de saúde mental ainda se encontra pouco presente, estando mais definida apenas no financiamento de programas destinados aos usuários de álcool e drogas.

Destaca-se, desde 2005, maior abertura da política para um conjunto de questões pertinentes à saúde mental (transexualismo, racismo, discriminação contra mulheres e outros grupos minoritários), as quais, até então, não encontravam espaço de discussão. A entrada desses temas promoveu maior articulação da saúde mental com diferentes áreas do Ministério da Saúde e também com áreas de outros ministérios e secretarias especiais.

\section{O papel dos estados nas macrofunções gestoras}

A atuação dos estados brasileiros na política de saúde mental é marcada pela heterogeneidade de fazeres e práticas. A apresentação dos resultados obtidos a partir das 24 coordenações estaduais está estruturada com base nas macrofunções gestoras em saúde (Tabela 3).

\section{Formulação de políticas e planejamento}

A maior parte das coordenações (19) está estruturada há mais de dez anos, o que indica tendência ao desenvolvimento de políticas de saúde mental nesses estados. Destacam-se como mais antigas as coordenações de Minas Gerais, Ceará e Mato Grosso. No conjunto, as coordenações são pequenas, apontando possíveis fragilidades em alguns estados na condução da política, mas, em alguns casos, podem indicar estratégia de fortalecimento dos municípios.

A maioria dos estados brasileiros possui plano de saúde mental em vigor, denotando certo esforço das coordenações no planejamento, especialmente a partir dos anos 2000, com a polí- tica de regionalização do sistema de saúde. Entretanto, embora as coordenações relatem a articulação da saúde mental com a regionalização, não fica claro como a primeira se insere nesse debate. Para muitos estados, subentende-se que a regionalização estaria ou associada à inserção das ações de saúde mental nas regiões de saúde, ou inserida nas propostas de regionalização da saúde prevista no plano estadual.

Ainda em relação ao plano, mais da metade das coordenações elabora seus planos em articulação com as diferentes áreas da secretaria, com as coordenações municipais, com colegiados estaduais e outras instâncias. Essa integração é de grande importância, não somente para o processo de formulação e planejamento da política, mas também para a coordenação federativa e da atenção. Todavia, foram identificadas poucas parcerias dos estados na elaboração de um plano regional de saúde mental.

Outro aspecto analisado foram as estratégias adotadas pelos estados de modo a atender às necessidades de cobertura dos serviços extrahospitalares nos seus municípios. As estratégias apontadas se resumiram à coordenação da pactuação da oferta de serviços com outros municípios, à ampliação das ações do programa de educação permanente e ao estímulo à formação de fóruns regionais de interlocução entre o estado e os municípios. No que se refere à estratégia de aporte de recursos financeiros estaduais para o arranjo de novos dispositivos assistenciais, esta foi identificada em 11 estados, com grande concentração nos estados das regiões Sul e Sudeste.

Quanto à avaliação da cobertura de CAPS nos estados, somente Alagoas, Sergipe e Paraná a consideraram suficiente. Quando questionados sobre os tipos de CAPS necessários, quase a totalidade dos estados (21) destacou a necessidade de se implantarem novos CAPS III, CAPSad e CAPSi (infanto-juvenil), mesmo naqueles estados que consideraram a cobertura destes serviços suficiente. No entanto, essa avaliação dos estados não coincide com a do Ministério da Saúde 16 , que considera a cobertura entre regular/baixa e insuficiente em apenas dez unidades da federação.

Logo, os estados apontam maior insuficiência de CAPS do que o Ministério, indicando que os parâmetros podem não estar adequados à realidade dos estados e municípios, além de mostrar fragilidade no planejamento integrado entre os três níveis.

\section{Coordenação federativa}

A função de coordenação federativa foi analisada verificando-se os arranjos adotados para a gestão 
O papel dos estados por macrofunções gestoras na política de saúde mental.

\begin{tabular}{lc}
\hline Macrofunções & Principais aspectos \\
\hline Formulação & Estruturação e características da \\
de políticas e & Coordenação de Saúde Mental; \\
planejamento & Elaboração de Plano de Saúde \\
Mental; Articulação do Plano com a & regionalização em saúde \\
foordenação & Arranjos adotados para a gestão em \\
saúde mental; Instrumentos legais e & normativos de pactuação e formalização \\
de compromissos; Mecanismos \\
e instrumentos utilizados para o \\
fortalecimento dos municípios
\end{tabular}

Coordenação da atenção

Financiamento

Monitoramento e acompanhamento

Prestação de serviços
Reestruturação da assistência em saúde mental; Mecanismos de organização do sistema de referência e contrarreferência entre serviços de saúde mental; Intersetorialidade; Participação social

Participação no financiamento do SUS Critérios utilizados para a alocação de recursos próprios destinados às ações de saúde mental; Promoção da PPI

Mecanismos e instrumentos utilizados para o acompanhamento e monitoramento dos serviços de saúde mental; Acompanhamento e avaliação de indicadores dos serviços de saúde mental

Prestação de ações e serviços, por meio de órgãos da Administração Direta ou Indireta vinculados à Secretaria Estadual

de Saúde, nos diversos campos da atenção à saúde mental

\section{Papel dos estados na saúde mental}

Balanço geral da atuação dos estados

A maior parte das coordenações possui, em média, mais de 10 anos de formação; Em 11 estados os primeiros planos de saúde mental foram apresentados nos anos 2000; Incipiente discussão sobre a política regional e a área de saúde mental

Grande interlocução com o CCSM; A maioria participa das CIBs quando o assunto em pauta refere-se à saúde mental; Pactuação de compromissos através de instrumentos legais envolvendo o processo de desinstitucionalização e financiamento em saúde mental; Padrão de atuação restrito à visita e supervisão dos municípios, participação em reuniões municipais e regionais, participação nas $\mathrm{CIBs}$ e promoção de discussões com os municípios sobre temas relevantes para o desenvolvimento da política

Liderança de alguns estados no processo de reestruturação da assistência psiquiátrica anterior ao PNASH; Concentração das centrais de regulação de internação psiquiátrica nos estados da Região Sudeste; Dificuldades para diminuir o número de reinternações; Articulação com diversos atores no desenvolvimento de projetos de saúde mental; Mais da metade dos estados desenvolvem ações que integram a comunidade na discussão da política de saúde mental

Fragilidade dos estados no financiamento em saúde mental

A maior parte das coordenações emite relatórios técnicos contendo o acompanhamento e monitoramento da política de saúde mental, embora com pouca divulgação às coordenações municipais; Principais tipos de acompanhamento e monitoramento: supervisão clínico-institucional, elaboração de diagnósticos; controle da distribuição de medicamentos psiquiátricos nos municípios; Pouca disponibilidade de profissionais, dificuldade dos CAPS de se constituir como um serviço de atenção à crise, modelo assistencial ainda reflete tendência à internação

A maior parte das coordenações possui a gestão de um serviço de saúde mental; Forte peso da gestão estadual de leitos SUS; Definição de serviços de saúde mental de iniciativa própria estadual em 13 estados
Em processo de consolidação

e amadurecimento da função

Em processo de consolidação e amadurecimento da função

Em processo de consolidação

e amadurecimento da função

Muito incipiente

Papel atuante no controle dos serviços

Papel atuante em alguns estados

CAPS: Centros de Atenção Psicossocial; CIB: Comissão Intergestores Bipartite; CCSM: Colegiado de Coordenadores de Saúde Mental; PNASH: Programa Nacional de Avaliação dos Serviços Hospitalares; PPI: Programação Pactuada e Integrada; SUS: Sistema Único de Saúde. Fonte: Elaborado a partir do questionário aplicado nos 24 estados e da revisão documental pertinente aos estados. 
em saúde, os instrumentos legais e normativos de pactuação e formalização de compromissos, além dos mecanismos e instrumentos utilizados para o fortalecimento dos municípios.

Em relação aos arranjos adotados para a gestão, verificou-se a participação dos estados no CCSM, na CIB e no Colegiado de Gestão Regional (CGR). Também procuramos identificar se os estados contavam com um Colegiado Estadual de coordenadores de saúde mental.

A maior parte das coordenações estaduais participa do CCSM, o qual passou a funcionar como um importante espaço de articulação federativa e fortaleceu a função de coordenação dos estados, garantindo maior integração e diálogo com o Ministério da Saúde e entre estados. Favoreceu, ainda, o debate sobre as estratégias de regionalização.

Em relação ao Colegiado Estadual de coordenadores, 11 estados afirmaram ter o colegiado. Para estes, as discussões travadas nesse âmbito contribuem para o encaminhamento das demandas dos municípios, principalmente para a discussão do plano municipal, pela troca de informações, pela busca de trabalho conjunto e formulação de propostas para o avanço da reforma.

No que diz respeito às CIBs, a maioria das coordenações faz parte desta comissão, mas os estados que participam o fazem em algumas reuniões, quando o assunto em pauta refere-se à saúde mental. Entre os principais assuntos abordados nas CIBs, destacam-se a discussão de incentivos financeiros para implantação de serviços substitutivos de saúde mental e a pactuação de metas para as regiões desse setor. Essa participação na CIB, quando o tema está em pauta, denota a forma de relação da saúde mental com a saúde pública.

Quanto à participação no CGR, metade das coordenações informou participar deste colegiado, e as que participam também o fazem em algumas reuniões, quando o assunto em pauta refere-se à saúde mental. Entretanto, deve-se considerar que nem todos os estados tinham CGR constituído no período de realização da pesquisa.

Em relação aos instrumentos legais e normativos de pactuação, verificou-se esforço de alguns estados na definição de novas leis e normativas inspiradas pelo PL da reforma. Ao longo da década de 1990, 7 estados (Rio Grande do Sul, Ceará, Pernambuco, Minas Gerais, Paraná, Rio Grande do Norte e Espírito Santo) e o Distrito Federal já haviam aprovado leis de saúde mental. No período de 1998 a 2009, 16 estados produziram normativas de saúde mental, entre leis, resoluções e portarias, prevendo a criação de colegiados de atenção nesse setor, comissões de revisão das internações involuntárias e de formação de Grupos de Trabalho para tratar da desinstitucionalização.

Dentre as normativas, destacam-se a Deliberação CIB $n^{\circ}$. 022/2009, de Santa Catarina, que aprova recursos de cofinanciamento da atenção básica para apoiar novos CAPS e CAPS microrregionais; a Deliberação CIB $n^{\circ}$. 023/2009, de Santa Catarina, que instituiu incentivo financeiro estadual aos Núcleos de Apoio à Saúde da Família (NASF) para municípios impossibilitados de cumprir os requisitos do Ministério da Saúde; o Decreto $n^{\circ}$. 12.573/2008, do Ministério da Saúde, sobre repasse de recursos do fundo estadual de saúde aos fundos municipais de saúde para a implementação de novos CAPS e adequação dos existentes. Tais documentos sugerem o esforço que os estados envolvidos na política de saúde mental fazem para que se garantam mecanismos de pactuação de compromissos, adotem-se arranjos de gestão regional e fortaleçam-se os municípios na gestão e prestação de serviços, principalmente por meio de apoio financeiro.

Identifica-se, ainda, que a coordenação federativa, com pactuação de compromissos, está bastante articulada a uma capacidade de financiamento dos estados, o que se torna um problema quando a unidade da federação não tem como propor incentivos.

Por fim, buscou-se reconhecer os mecanismos e instrumentos utilizados pelos estados para fortalecer os municípios. Verificou-se um padrão de atuação restrito à visita e supervisão dos municípios, participação em reuniões municipais e regionais, participação nas CIBs e promoção de discussões com os municípios sobre temas relevantes para o desenvolvimento da política. A relação dos estados com os municípios é bastante controversa, sugerindo um padrão de hierarquia administrativa que se sobrepõe a qualquer prática de diálogo ou busca de parceria.

A política de educação permanente foi pouco indicada pelas coordenações, apesar da política de incentivos para formação e qualificação promovida pelo Ministério da Saúde desde 2003 e da queixa de muitos estados em relação à qualificação de profissionais para atuar na atenção.

Quando o assunto se refere ao tipo de apoio prestado pelos estados para o desenvolvimento de uma política nos municípios com menos de 20 mil habitantes, o financiamento das ações de saúde mental na atenção básica é indicado na resposta de mais da metade dos estados. Além desse tipo de apoio, também foram identificadas estratégias de implementação de equipes matriciais de referência, coordenação da articulação com os municípios numa política regional e formação de recursos humanos. 
Talvez contribua para este fato a ênfase que vem sendo dada pelo governo federal ao desenvolvimento de uma política de atenção básica, principalmente por intermédio dos NASF e do Programa Saúde da Família (PSF). Já ressaltamos a importância do papel dos estados no desenvolvimento de uma política de saúde mental nos municípios que, não podendo contar com um CAPS, buscam a articulação das ações de saúde mental na atenção básica.

\section{Coordenação da atenção}

A coordenação da atenção foi analisada de acordo com os mecanismos e instrumentos utilizados para a integração e organização da assistência no território. Entre eles, destacam-se: reestruturação da assistência, implantação de mecanismos de referência e contrarreferência entre serviços, intersetorialidade e participação social.

Em relação à reestruturação da assistência, dez estados afirmaram ter liderado um processo de reestruturação dos hospitais psiquiátricos, especialmente por meio da avaliação da qualidade do atendimento hospitalar e da redução do porte dos hospitais. Esse processo foi anterior à implementação dos Programas de Avaliação e Reestruturação Hospitalar promovidos pelo Ministério (PNASH/2002 e PRH/2004), mas, para a maioria dos estados, a apresentação desses programas possibilitou a definição de mudanças na política para os macro-hospitais, principalmente por ter orientado uma ação nesta área e por possibilitar a criação de novos incentivos extra-hospitalares. Contudo, para alguns estados, essas mudanças ainda não são suficientes.

No que diz respeito aos mecanismos de organização do sistema, somente seis estados afirmaram possuir uma central de regulação de internação psiquiátrica (Roraima, Rio Grande do Norte, Minas Gerais, Rio de Janeiro, São Paulo e Paraná). Verifica-se que as centrais estão concentradas no Sudeste, que, historicamente, abriga um grande parque manicomial. As centrais surgiram na década de 2000, período em que os estados passaram a assumir maior importância na regionalização da saúde.

Entre as maiores dificuldades apontadas pelos estados para diminuir o número de reinternações, estão o número de serviços extra-hospitalares menores que a demanda e a dificuldade de o CAPS se constituir como um serviço de atenção à crise. Alguns estados destacaram outros fatores, como a não continuidade do atendimento extrahospitalar por parte do paciente e da família; a relutância da atenção básica em atender os pacientes e/ou encaminhá-los ao CAPS e ambulatórios; a falta de leitos de curta duração para urgência; o número excessivo de leitos contratados; e a forte influência de entidades ligadas às categorias médica e hospitalar, constituindo um movimento de resistência à reforma psiquiátrica.

Em relação à intersetorialidade, observouse a existência, em todos os estados, de áreas da Secretaria Estadual parceiras da coordenação de saúde mental, como a atenção básica, DST/AIDS, vigilância epidemiológica, saúde do trabalhador, saúde da criança, saúde da mulher, saúde bucal, entre outras. Na maior parte dos estados, existem projetos e parcerias da coordenação com outras secretarias estaduais e demais entidades (universidades, ONGs, Tribunal de Justiça e Ministério Público).

No que diz respeito à participação social, verificamos que, somente em sete estados, o conselho estadual de saúde conta com uma comissão estadual de saúde mental. Este fato é esperado, visto que a formação de uma comissão específica no interior do conselho de saúde é facultativa, embora a sua criação indique articulação do estado com outros atores envolvidos no desenvolvimento de uma política de saúde mental.

Mais da metade dos estados afirmaram desenvolver ações que integram a comunidade na discussão da política de saúde mental. Tal integração se dá por meio da articulação com conselhos estaduais e municipais e com o colegiado de políticas de atenção psicossocial. Há, ainda, a promoção de eventos abertos de capacitação e encontros anuais de profissionais, familiares $\mathrm{e}$ usuários.

\section{Financiamento}

A função de financiamento foi analisada verificando-se a definição de percentual mínimo do orçamento da saúde para o financiamento da área, os critérios utilizados para a alocação de recursos próprios destinados às ações de saúde mental e a promoção da programação pactuada e integrada da assistência à saúde (PPI).

Somente oito estados definem percentual de recursos da saúde para o financiamento das ações de saúde mental. Já a definição de critérios para a transferência de recursos próprios no financiamento das ações foi indicada por 12 estados. Dentre os critérios utilizados, estão: municípios mais populosos; municípios de fácil acesso geográfico e com unidades psiquiátricas desativadas; municípios com carência de serviços e ações de saúde mental; municípios com dificuldade de custear serviços extra-hospitalares. Igualmente em 12 estados, os recursos financeiros federais destinados às ações de saúde mental estão previstos na PPI, mediante a celebração de convênios, de repasse de recursos 
para os municípios que não estão no exercício pleno da gestão, da pactuação de serviços extrahospitalares e de repasse de recursos para a implementação de CAPS.

Apesar de as normativas e diretrizes da política de saúde mental destacarem a necessidade do repasse de recursos financeiros das secretarias estaduais aos municípios e de definir um percentual mínimo nos respectivos orçamentos anuais e plurianuais para a saúde mental, esta é uma função ainda frágil no âmbito dos estados. A discussão do financiamento surge mais incisivamente no momento de pactuação para distribuição dos recursos destinados à área, mas não garante a entrada de recursos novos. Esta é uma questão para os estados e para o gestor federal, que também enfrenta dificuldades para garantir recursos para o setor.

\section{Acompanhamento e monitoramento}

Esta função foi analisada conforme os mecanismos, instrumentos e indicadores utilizados para o acompanhamento e monitoramento dos serviços. Entre os principais tipos de acompanhamento e monitoramento exercidos pelos estados, destacam-se a supervisão clínico-institucional dos serviços de saúde mental, a elaboração de diagnósticos e o controle da distribuição de medicamentos psiquiátricos nos municípios.

No que diz respeito à avaliação dos serviços de saúde mental nos estados, foram feitas perguntas abertas com relação à situação das instalações físicas, disponibilidade de profissionais, modelo assistencial, atendimento à crise e clientela dos CAPS.

Em relação às instalações físicas, 13 estados apontaram condições regulares e adequadas dos CAPS, sete indicaram que as instalações encontram-se precárias e improvisadas e dois relataram possuir realidades distintas, ou seja, CAPS com instalações adequadas e serviços em condições ruins.

Quanto à disponibilidade de profissionais, 16 estados a consideraram precária, pela dificuldade de contratação de psiquiatras, pela grande rotatividade de técnicos sem vínculo efetivo e pelo fato de a maioria dos CAPS possuir somente a equipe mínima, o que é insuficiente para o atendimento da demanda.

Com relação ao atendimento à crise, 19 estados apontaram a dificuldade dos CAPS de se constituir como um serviço de atenção à crise, principalmente pela falta de preparo dos profissionais. Esse quadro leva ao encaminhamento dos casos para os hospitais psiquiátricos.

Com relação ao modelo assistencial, para 15 estados o modelo ainda reflete uma tendência à internação, estando também centrado no atendimento ambulatorial e medicamentoso, além de apresentar dificuldades de articulação do trabalho em rede. Foi mencionado que os CAPS do estado funcionariam como uma espécie de serviço "rígido", que não atenderia certos tipos de clientela, selecionando-as e encaminhando casos de crise aos prontos-socorros.

Já em relação à clientela, as respostas foram bastante variadas, mas muitos indicaram a presença de demanda reprimida, principalmente no atendimento a usuários de álcool e drogas.

Os estados retratam uma situação crítica na organização da atenção em saúde mental substitutiva, indicando elementos importantes que precisam ser enfrentados. Ressalte-se que a maior parte das coordenações emite relatórios técnicos da política em seu estado, porém nem todas divulgam os resultados desses relatórios às coordenações dos seus municípios.

\section{Prestação de serviços}

A função de prestação refere-se à gestão e definição de serviços de saúde mental de iniciativa própria.

A maior parte das coordenações é responsável pela gestão de um serviço, principalmente de hospitais psiquiátricos, ambulatórios e CAPSad. Também 9 estados disseram ter assumido a gestão de um determinado serviço de saúde mental por situação de carência ou omissão por parte do município. Esse quadro se deu, sobretudo, nas regiões Norte e Nordeste, indicando a importância estratégica dos estados enquanto atores responsáveis pela prestação de serviços quando da ausência e/ou incapacidade municipal.

Verifica-se, ainda, o forte peso da gestão estadual de leitos SUS psiquiátricos, quando comparados com o número de leitos de gestão municipal e federal. Destacam-se os estados de Minas Gerais, Pernambuco, Rio de Janeiro e São Paulo (Tabela 4) 17. Em adição, se o número de leitos SUS sob gestão estadual é grande nestes estados, o número de leitos psiquiátricos privados existentes é ainda maior, o que os torna os estados com o maior número de leitos psiquiátricos totais do país, incluindo os de gestão municipal, estadual e federal vinculados ao SUS, e os privados conveniados ao SUS.

Deve-se ressaltar que o peso da iniciativa privada no sistema de saúde brasileiro, principalmente nas regiões Sul e Sudeste, é ainda importante, indicando a fragilidade do poder público na assistência em saúde mental, sendo este um desafio importante para a reforma.

Esses dados ratificam a importância dos estados no planejamento da rede de saúde mental e 
Número de leitos psiquiátricos por esfera administrativa *. Brasil, 2010.

\begin{tabular}{|c|c|c|c|c|c|c|}
\hline Região/Estados & Federal (SUS) & $\begin{array}{c}\text { Estadual } \\
\text { (SUS) }\end{array}$ & $\begin{array}{c}\text { Municipal } \\
\text { (SUS) }\end{array}$ & $\begin{array}{c}\text { Privados } \\
\text { (conveniados } \\
\text { SUS) }\end{array}$ & $\begin{array}{c}\text { Privados } \\
\text { (existentes) }\end{array}$ & Leitos totais \\
\hline \multicolumn{7}{|l|}{ Norte } \\
\hline$A C$ & 0 & 71 & 2 & 1 & 2 & 75 \\
\hline AM & 0 & 126 & 1 & 0 & 2 & 129 \\
\hline AP & 0 & 16 & 0 & 0 & 0 & 16 \\
\hline PA & 4 & 121 & 10 & 3 & 45 & 182 \\
\hline $\mathrm{RR}$ & 0 & 12 & 0 & 0 & 0 & 12 \\
\hline $\mathrm{RO}$ & 0 & 35 & 0 & 0 & 4 & 40 \\
\hline TO & 0 & 10 & 3 & 160 & 170 & 183 \\
\hline \multicolumn{7}{|l|}{ Nordeste } \\
\hline$A L$ & 0 & 160 & 0 & 720 & 840 & 1.000 \\
\hline MA & 0 & 108 & 104 & 560 & 697 & 910 \\
\hline PB & 0 & 244 & 32 & 837 & 1.005 & 1.281 \\
\hline $\mathrm{PE}$ & 12 & 795 & 9 & 2.805 & 3.048 & 3.865 \\
\hline $\mathrm{PI}$ & 0 & 166 & 7 & 223 & 245 & 430 \\
\hline $\mathrm{RN}$ & 3 & 158 & 174 & 367 & 480 & 841 \\
\hline SE & 8 & 0 & 5 & 296 & 368 & 381 \\
\hline BA & 10 & 640 & 25 & 868 & 1057 & 1.732 \\
\hline CE & 2 & 170 & 22 & 955 & 1.097 & 1.291 \\
\hline \multicolumn{7}{|l|}{ Centro-oeste } \\
\hline DF & 2 & 109 & 0 & 0 & 414 & 526 \\
\hline GO & 15 & 15 & 43 & 1.255 & 1787 & 1.860 \\
\hline MS & 4 & 10 & 9 & 295 & 418 & 441 \\
\hline $\mathrm{MT}$ & 0 & 122 & 7 & 84 & 86 & 215 \\
\hline \multicolumn{7}{|l|}{ Sudeste } \\
\hline MG & 39 & 546 & 25 & 2.096 & 3.635 & 4.245 \\
\hline RJ & 124 & 812 & 1.353 & 4.788 & 6.800 & 9.232 \\
\hline $\mathrm{SP}$ & 16 & 2.507 & 255 & 9.832 & 12.452 & 15.266 \\
\hline ES & 0 & 241 & 1 & 408 & 587 & 829 \\
\hline \multicolumn{7}{|l|}{ Sul } \\
\hline PR & 0 & 244 & 31 & 2.348 & 3.019 & 3.296 \\
\hline RS & 76 & 200 & 127 & 1.561 & 2.440 & 2.873 \\
\hline SC & 0 & 475 & 35 & 641 & 940 & 1.450 \\
\hline
\end{tabular}

* É importante destacar que os dados do CNES, disponíveis na Sala de Situação em Saúde do Ministério da Saúde, apresentam valores divergentes quanto ao número real de leitos psiquiátricos por esfera administrativa nos estados. Estados: AC: Acre; AL: Alagoas; AM: Amazonas; AP: Amapá; BA: Bahia; CE: Ceará; DF: Distrito Federal; ES: Espírito Santo; GO: Goiás; MA: Maranhão; MG: Minas Gerais; MS: Mato Grosso do Sul; MT: Mato Grosso; PA: Pará; PB: Paraíba; PE: Pernambuco; PI: Piauí; PR: Paraná; RJ: Rio de Janeiro; RN: Rio Grande do Norte; RO: Rondônia; RR: Roraima; RS: Rio Grande do Sul; SC: Santa Catarina; SE: Sergipe; SP: São Paulo; TO: Tocantins.

Fonte: Ministério da Saúde 17.

na articulação com os municípios, conduzindo a desospitalizações psiquiátricas e garantindo cuidados extra-hospitalares aos pacientes em processo de desinstitucionalização. Indicam, também, a necessidade de continuidade do processo de reestruturação da assistência psiquiátrica e de redução do número de leitos em hospitais psiquiátricos no país, embora, em alguns estados, já se verifique o esforço no desenvolvimento de ações nesta área.

A definição de serviços de saúde mental de iniciativa estadual foi verificada em 13 estados, 
demonstrando o protagonismo e a autonomia destes na criação de modelos que visem a atender às especificidades e necessidades de seus territórios. Dentre esses modelos, destacam-se o Programa Vida em Casa, do Distrito Federal, que oferece a pacientes com mais de dois anos de transtorno psicótico grave e de família carente o atendimento residencial na fase aguda do transtorno; o Programa Saúde Mais Perto de Você, do Rio Grande do Sul, que destina recursos financeiros para leitos psiquiátricos e de dependência química em hospitais gerais.

Foram identificadas experiências igualmente exitosas em diferentes estados, como o fechamento do Hospital Estadual Teixeira Brandão e a implantação de 28 serviços residenciais terapêuticos (SRTs) no Município de Carmo (Rio de Janeiro). Somem-se a estas o fechamento do Hospital Psiquiátrico em Caicó (Rio Grande do Norte) e sua transformação em CAPS III.

\section{Considerações finais}

O estudo do papel dos estados na política de saúde mental permitiu que se aprofundasse a compreensão sobre como os estados têm lidado com os dilemas da gestão nesta área e quais as potencialidades e limites desta condução.

Observamos um esforço recente de um conjunto expressivo de estados na elaboração de seus planos de saúde mental e de articulação com a regionalização. Os anos 2000 foram de expansão das políticas em todo território, exigindo das unidades federativas papel mais ativo na formulação e planejamento das políticas. Verificamos, ainda, empenho dos estados na construção de marcos legais e na articulação com o Ministério da Saúde e municípios, principalmente por meio da participação ativa nos fóruns de deliberação da política.

O esforço de coordenação federativa também levou a um esforço de organização da atenção, mas ainda se mostram necessárias ações que visem à integração da assistência no território, assegurando o atendimento e cuidado em saúde mental na rede extra-hospitalar. Além disso, apesar da importância do CAPS na reorganização da assistência no território, ainda existem barreiras que o impedem de se constituir como um serviço de atenção à crise.

Os estados exercem papel atuante no acompanhamento e monitoramento dos municípios, especialmente no controle dos serviços, mas não foram apresentados mecanismos para compartilhamento desse controle, muito menos de qualificação dos profissionais. O relato dos estados é de uma situação bastante precária na atenção, havendo dificuldades para se empreender uma mudança no cenário assistencial.

Muitos estados têm assumido seu papel de executores de serviços de saúde mental, não apenas naqueles voltados ao atendimento de usuários de álcool e drogas, mas também na gestão de leitos psiquiátricos, além de desenvolverem iniciativas e experiências exitosas na área. Ainda assim, não há indícios de uma postura ativa dos estados na criação de modelos mais próprios.

A função de financiamento é a mais frágil de todas, comprometendo qualquer projeto de mudança mais contundente no desenho do modelo assistencial. A saúde mental encontra dificuldades importantes para garantir incentivos e recursos novos no contexto do SUS. A análise do papel dos estados permite inferir que a superação dos desafios relacionados ao processo de condução estadual da política de saúde mental depende da articulação entre governo federal, estados e municípios na elaboração de políticas que atendam à especificidade de cada região, macro ou micro, e da promoção de um planejamento ascendente e participativo.

Estados que possuem maior poder de indução política dentro de uma determinada região, principalmente nas regiões Norte, Centro-Oeste e Nordeste - onde existe maior carência de profissionais e serviços - podem realizar parcerias para a elaboração de planos de investimentos e de desenvolvimento institucional com os estados e municípios menos favorecidos da região. Também é necessário o desenvolvimento de uma política de investimentos para o setor que favoreça um papel mais ativo dos estados no financiamento em saúde mental, possibilitando investimentos na rede extra-hospitalar e a pactuação de novos compromissos para o desenvolvimento da política.

Finalmente, deve-se superar a existência da cultura institucional que gera dificuldades para a formulação do modelo assistencial, sendo necessário seu enfrentamento para a continuidade de uma reforma compromissada com os princípios do SUS e de uma assistência humanizada aos usuários dos serviços de saúde mental. 


\section{Resumo}

Este artigo analisa o papel dos estados na política de saúde mental no Brasil no período de 1990 a 2009. A metodologia incluiu a aplicação de questionário eletrônico nas coordenações estaduais de saúde mental de 24 estados brasileiros, revisão documental e análise de base de dados oficiais. Os resultados obtidos apontaram que os estados utilizam várias estratégias e instrumentos na condução da política de saúde mental, principalmente no monitoramento e na prestação de serviços. No entanto, percebeu-se que os estados ainda não desenvolvem de forma sistemática as funções de planejamento, coordenação federativa e coordenação da atenção. O financiamento em saúde mental representa um dos grandes desafios para a gestão dessa área nos estados. Os achados deste estudo sugerem que a superação dos desafios relacionados à condução estadual da política de saúde mental depende da articulação entre governo federal, estados e municípios na elaboração de políticas que atendam à especificidade de cada região, da promoção de um planejamento participativo e de investimentos para o setor.

Saúde Mental; Política de Saúde; Gestão em Saúde

\section{Colaboradores}

A. G. Simon e T. W. F. Baptista participaram igualmente da produção do artigo.

\section{Referências}

1. Brasil. Lei no ${ }^{\circ}$. 8080, de 19 de setembro de 1990. Lei orgânica da saúde. Diário Oficial da União 1990; 20 set.

2. Lima LD, Machado CV, Baptista TWF, Pereira AM. O Pacto Federativo brasileiro e o papel do gestor estadual no SUS. In: Ugá MAD, Sá MC, Martins M, Braga Neto FC, organizadores. A gestão do SUS no âmbito estadual: o caso do Rio de Janeiro. Rio de Janeiro: Editora Fiocruz; 2010. p. 27-58.

3. Borges CF, Baptista TWF. O modelo assistencial em saúde mental: a trajetória da construção política de 1990 a 2004. Cad Saúde Pública 2008; 24:456-68.

4. Brasil. Lei no. 10.216, de 6 de abril de 2001. Dispõe sobre a proteção e os direitos das pessoas portadoras de transtornos mentais e redireciona o modelo assistencial em saúde mental. Diário Oficial da União 2011; 9 abr.

5. Departamento de Assistência e Promoção à Saúde, Secretaria de Assistência à Saúde, Ministério da Saúde. Relatório final da II Conferência Nacional de Saúde Mental. Brasília: Ministério da Saúde; 1994.
6. Thornicroft G, Alem A, Antunes dos Santos R, Barley E, Drake RE, Gregorio G, et al. WPA guidance on steps, obstacles and mistakes to avoid in the implementation of community mental health care. World Psychiatry 2010; 9:67-77.

7. Patel V, Araya R, Chatterjee S, Chisholm D, Cohen A, De Silva M, et al. Treatment and prevention of mental disorders in low-income and middle-income countries. Lancet 2007; 370:991-1005.

8. Thornicroft G, Tansella M. Components of a modern mental health service: a pragmatic balance of community and hospital care: overview of systematic evidence. Br J Psychiatr 2004; 185:283-90.

9. Lancet Global Mental Health Group; Chisholm D, Flisher AJ, Lund C, Patel V, Saxena S, et al. Scale up services for mental disorders: a call for action. Lancet 2007; 370:1241-52.

10. Lima LD. Estados federativos e políticas públicas. In: Lima LD, organizador. Federalismo, relações fiscais e financiamento do Sistema Único de Saúde: a distribuição de receitas vinculadas à saúde nos orçamentos municipais e estaduais. Rio de Janeiro: Museu da República; 2007. p. 31-60. 
11. Machado CV. O modelo de intervenção do Ministério da Saúde brasileiro nos anos 90. Cad Saúde Pública 2007; 23:2113-26.

12. Mendes EV. Os sistemas de serviços de saúde: o que os gestores deveriam saber sobre essas organizações complexas. Fortaleza: Escola de Saúde Pública do Ceará; 2002.

13. Lima LD, Kuschnir R, Pereira AM. Análise de experiências estaduais em curso de regionalização e construção de redes de serviços de saúde. Relatório final. Rio de Janeiro: Escola Nacional de Saúde Pública Sergio Arouca, Fundação Oswaldo Cruz/ Secretaria de Estado da Saúde e Defesa Civil; 2009.

14. Borges CF. Políticas de saúde mental e sua inserção no SUS: a discussão de convergências e divergências e o resgate de alguns conceitos e valores pertinentes à reforma psiquiátrica [Dissertação de Mestrado]. Rio de Janeiro: Escola Nacional de Saúde Pública Sergio Arouca, Fundação Oswaldo Cruz; 2007.
15. Secretaria de Atenção à Saúde, Ministério da Saúde. Saúde mental no SUS: acesso ao tratamento e mudança do modelo de atenção. Relatório de gestão 2003-2006. Brasília: Ministério da Saúde; 2007.

16. Coordenação Geral de Saúde Mental, Departamento de Ações Programáticas Estratégicas, Secretaria de Atenção à Saúde, Ministério da Saúde/ Instituto Brasileiro de Geografia e Estatística. Cobertura de CAPS, por UF (junho 2009) e estimativa populacional em 2008. Brasília: Ministério da Saúde; 2009.

17. Sala de Situação em Saúde, Ministério da Saúde. Gestão em saúde - estabelecimentos/CNES, 2010. http://www.datasus.gov.br/rnis/saladesituacao. htm (acessado em 05/Abr/2010).

Recebido em 18/Jan/2011

Versão final reapresentada em 09/Mai/2011

Aprovado em 19/Ago/2011 